\title{
Temporal Fluctuations in a Turbulently Stirred Binary Liquid Mixture
}

\author{
P. Tong, ${ }^{(1,2)}$ W. I. Goldburg, ${ }^{(1)}$ J. Stavans, ${ }^{(1),(a)}$ and A. Onuki ${ }^{(3)}$ \\ ${ }^{(1)}$ Department of Physics and Astronomy, University of Pittsburgh, Pittsburgh, Pennsylvania 15260 \\ ${ }^{(2)}$ Exxon Research and Engineering Company, Annandale, New Jersey 08801 \\ ${ }^{(3)}$ Research Institute for Fundamental Physics, Kyoto University, Kyoto 606, Japan \\ (Received 8 March 1989)
}

\begin{abstract}
We report measurements of the temporal autocorrelation function $g(t)$ of light scattered from critical binary liquid mixtures which are being continuously and turbulently stirred. The functional form $g(t)$ changes dramatically when the turbulent shear is changed from weak to strong. In the strong shear regime $g(t)$ becomes a stretched exponential function, with an exponent that decreases as the temperature approaches the critical temperature. This stretched exponential behavior is associated with spatially intermittent suppression of critical fluctuations produced by the turbulence.
\end{abstract}

PACS numbers: $42.20 .-\mathrm{y}, 05.70 . \mathrm{Jk}, 47.25 . \mathrm{Mr}$

It is well known that in fluid mixtures near the critical point, the local composition fluctuates strongly about its mean value, and the lifetime of these fluctuations becomes very long.' If the mixture is vigorously stirred, the equilibrium spectrum of thermally excited fluctuations will be strongly modified by the local velocity gradient, $S(\mathbf{r})$. We have used photon correlation homodyne spectroscopy ${ }^{2}$ to study the effect of this stirring on temporal fluctuations in a critical binary liquid mixture. We observe that when the mixture is far from the critical point, where the shear is "weak," the measured intensity correlation function $g(t)$ becomes independent of the temperature, and is controlled by the flow field. Near the critical point, the effect of the random shear is more dramatic. The measured $g(t)$ is found to be temperature dependent, and can be well fitted by a stretched exponential function.

To judge the effect of the random shear $S(\mathbf{r})$ on the composition fluctuations $\chi_{k}=\langle|c(\mathbf{k}, t) c(-\mathbf{k}, t)|\rangle$ $[c(\mathbf{k}, t)$ being the Fourier component of the composition deviation $\delta c(\mathbf{r}, t)$ from its mean value], it is necessary to compare the shear rate $S(\mathbf{r})$ with the relaxation rate, $\Gamma_{\xi}=\left(k_{B} T / 6 \pi \eta\right) \xi^{-3}$, of equilibrium composition fluctuations. ${ }^{3}$ Here $\eta$ is the viscosity of the mixture and $\xi=\xi_{0}\left|T / T_{c}-1\right|^{-0.625}$ is the thermal correlation length. When $S(\mathbf{r})$ is small compared to $\Gamma_{\xi}$, the fluctuations appear and disappear in a time shorter than $[S(\mathbf{r})]^{-1}$, and hence the effect of $S(\mathbf{r})$ on local composition will be small (weak shear region). In the opposite limit of strong shear, where $S(\mathbf{r}) \gg \Gamma_{\xi}$, the shear will stretch and thereby suppress the composition fluctuation $\delta c(\mathbf{r}, t)$. This stretching drastically alters the angular distribution of the scattered light. When the shear is uniform, both theory ${ }^{4}$ and experiment ${ }^{5}$ have shown that the strong shear produces a weak but measurable shift in the critical temperature $T_{c}$, and changes critical exponents. The effect of turbulent shear on the wave-number spectrum of composition fluctuations has also been studied, ${ }^{6}$ but there are no prior studies of the effect of turbulent stir- ring on temporal fluctuations in a binary mixture

We report here measurements of the temporal autocorrelation function, $g(t)=\langle I(0) I(t)\rangle$, of scattered light intensity $I(t)$ at scattering vector $\mathbf{k}$. The critical binary mixtures studied in the experiment were isobutyric acid plus water (IBW) and 2,6-lutidine plus water (LW). The experiment was performed in the one-phase region of the mixtures, and the control parameters were the temperature difference $\left|T-T_{c}\right|$ and the stirring frequency $f$. The experimental arrangement is similar to that of Ref. 6. The critical mixtures were sealed in cylindrically shaped Pyrex cells, $\sim 8 \mathrm{~cm}$ in height and $4 \mathrm{~cm}$ in diameter. Turbulence was generated with a magnetically driven stirrer contained within the sample cell. The stirring bar was $0.9 \mathrm{~cm}$ in diameter and $2.5 \mathrm{~cm}$ in length. Each sample was located in a water bath in which the temperature was controlled within $10 \mathrm{mK}$ during a run. The sample cell also contained a thermistor to monitor the temperature of the mixture in the cell. The turbulence-generated heating raised the internal temperature of the mixture to about $13 \mathrm{mK}$ above the bath temperature at $f=10 \mathrm{~Hz}$.

The beam from a He-Ne laser traversed the cell $2 \mathrm{~cm}$ above the stirring bar, where the turbulence was strongest. The light intensity scattered by the mixture was collected by a photomultiplier. Measurements were made at two scattering angles, $60^{\circ}$ and $90^{\circ}$. The scattering vector $k=(4 \pi n / \lambda) \sin (\theta / 2)$, where $\lambda(=488 \mathrm{~nm})$ is the wavelength of the incident light and $n(=1.3)$ is the refractive index. Using a digital correlator we measure the homodyne autocorrelation function $g(t)=\langle I(0) I(t)\rangle$ $=\left\langle I^{2}\right\rangle[1+A G(t)]$, where $A$ is a constant which depends on the geometry of the experimental setup.

In our experiment the stirring frequency $f$ ranged from 5 to $14 \mathrm{~Hz}$. The corresponding Reynolds number $\mathrm{Re}=2 \pi f r^{2} / v$ is the range $1.7 \times 10^{3}$ to $4.7 \times 10^{3}$. In the above $r(=1.25 \mathrm{~cm})_{\mathrm{O}}$ is the radius of the stirrer, and $v$ $\left(=0.33 \mathrm{~cm}^{2} / \mathrm{sec}\right.$ for IBW) is the kinematic viscosity of the mixture. Since the turbulence was very far from ful- 
ly developed, ${ }^{6}$ a more realistic maximum value of $\mathrm{Re}$ might better be taken as $10^{3}$. To have an order-ofmagnitude estimate for some characteristic parameters in our turbulent flow, we invoke the Kolmogorov theory of fully developed turbulence because no other theory is available at this point for flows with the abovementioned Reynolds number. From the Kolmogorov view of turbulence, ${ }^{7}$ the kinetic energy of turbulence is continuously transferred from large scales down to smaller scales until dissipation occurs. In our case continuously stirring the mixture generates eddies of size $l_{0}$ $(=2.5 \mathrm{~cm}$, the size of the stirrer $)$, and causes the large eddies to break up into smaller and smaller ones, of size $l_{0} / 2, l_{0} / 2^{2}$, etc., until finally a smallest size $l_{d}=l_{0} \mathrm{Re}^{-3 / 4}$ is reached. In the inertial range $l_{d} \ll l \ll l_{0}$, the cascade proceeds without loss of energy into heat. From a simple dimensional argument the characteristic velocity $u(l)$ associated with eddies of size $l$ has the scaling form $u(l) \simeq(\epsilon l)^{1 / 3}$, where $\epsilon$ is the energy injection rate. The maximum turbulent shear rate is $S\left(l_{d}\right) \simeq v \mathrm{Re}^{3 / 2} / l_{0}^{2}$. This gives $S\left(l_{d}\right)=150 \mathrm{sec}^{-1}$ and $l_{d} \simeq 0.1 \mathrm{~mm}$ when $\mathrm{Re}=10^{3}$. One can determine the strong shear region in terms of the length scale $\xi_{c}$ by equating $S\left(l_{d}\right)$ to $\Gamma_{\xi}=\xi_{c}$. This also defines a temperature difference $\Delta T$, using $\xi_{c}=\xi_{0}\left|\Delta T / T_{c}\right|^{-0.625}$. For $\operatorname{Re}=10^{3}$ we have $\xi_{c}=8$ $\times 10^{-6} \mathrm{~cm}$ and $\Delta T=53 \mathrm{mK}$.

We first describe measurements made in the one-phase region far from $T_{c}$ in the critical $\mathrm{LW}$ mixture, which has an inverted coexistence curve with $T_{c}=33.37^{\circ} \mathrm{C}$. Figure 1 shows the measured $G(t)$ (crosses) at $T_{c}-T=170$ $\mathrm{mK} \gg \Delta T$. The diamonds in Fig. 1 are the measurements made in the identical cell, but with the LW mixture replaced with water seeded with polystyrene spheres of diameter $0.06 \mu \mathrm{m}$. The agreement between these two sets of measurements, and the added fact that $G(t)$ is

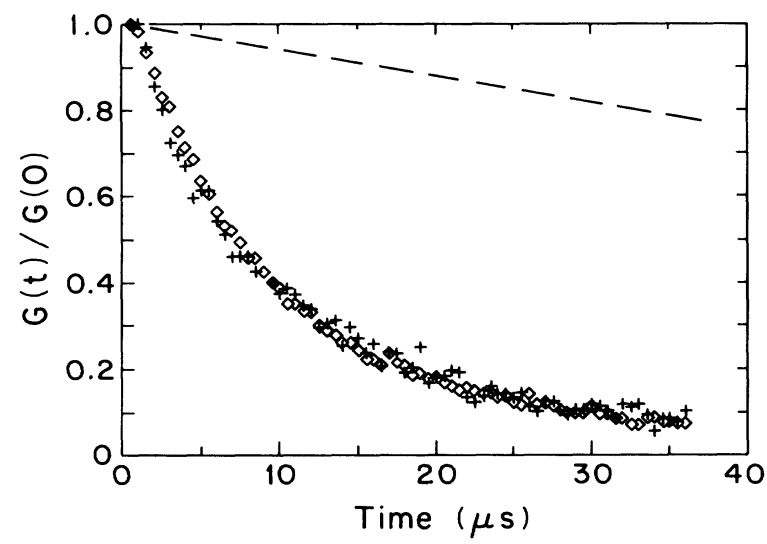

FIG. 1. The normalized $G(t)$ for $\mathrm{LW}$ at $T_{c}-T=170 \mathrm{mK}$ $(x)$, and for a weak aqueous solution of polystyrene spheres of diameter $0.06 \mu \mathrm{m}(\diamond)$. The two sets of measurements were made in containers of identical shape with $f=8 \mathrm{~Hz}$ and $\theta=90^{\circ}$. The dashed line shows $G(t)$ in $\mathrm{LW}$ at equilibrium $(f=0 \mathrm{~Hz})$ and $T_{c}-T=170 \mathrm{mK}$. temperature dependent when $\left|T-T_{c}\right| \gg \Delta T$ (see Fig. 2 ), confirms that in the weak shear region $G(t)$ is dictated by the turbulent velocity field. For comparison we also include in Fig. 1 the measured $G(t)$ in LW in its equilibrium state, i.e., when $f=0 \mathrm{~Hz}$ (dashed line). In this case $G(t)$ decays exponentially with the rate $2 D k^{2}$, where $D=k_{B} T / 6 \pi \eta \xi$ is the diffusivity of the mixture. We will show below that the decay time $G(t)$ in the weak shear regime is proportional to $[k u(L)]^{-1}$, where $L$ is the width of a slit, which controls the size of the scattering volume viewed by the photodetector. For all the measurements presented in this Letter, $L=1 \mathrm{~mm}$, which is much greater than the beam diameter $d$ $(d<0.1 \mathrm{~mm})$. From Fig. 1 it is seen that the decay time of $G(t)$ is about $8 \mu \mathrm{sec}$ when $f=8 \mathrm{~Hz}$, from which we have $u(L)=0.5 \mathrm{~cm} / \mathrm{sec}$, and $S(L)=u(L) / L \simeq 5 \mathrm{sec}^{-1}$.

We turn next to the measurements carried out in the IBW mixture, which has a normal coexistence curve with $T_{c}=26.43^{\circ} \mathrm{C}$. This mixture scatters light less than $\mathrm{LW}$, so that one can avoid the multiple scattering near the critical point. The data in Fig. 2 illustrate a feature shared by all our measurements: The functional form and decay time of $G(t)$, at fixed stirring frequency and scattering angle, is the same when the temperature is sufficiently far from $T_{c}$, i.e., when $\left|T-T_{c}\right| \gg \Delta T$. Two examples with $T-T_{c}=700 \mathrm{mK}$ and $T-T_{c}=305 \mathrm{mK}$ are shown in Fig. 2 (crosses and diamonds). However, when $T-T_{c} \leq \Delta T=50 \mathrm{mK}$, we observe that the functional form of $G(t)$ changes dramatically. This is clearly seen in Fig. 2 where a measured $G(t)$ at $T-T_{c}=10 \mathrm{mK}$ is plotted (squares). Note the very sharp decay $G(t)$ at small time $t$. We should mention that the temperatures quoted here have not been corrected for the turbulent heating, which is $\sim 13 \mathrm{mK}$.

In the temperature range $10 \mathrm{mK} \leq T-T_{c} \leq 50 \mathrm{mK}$ and at various stirring frequencies, it is found that the measured $G(t)$ can be well fitted by the stretched ex-

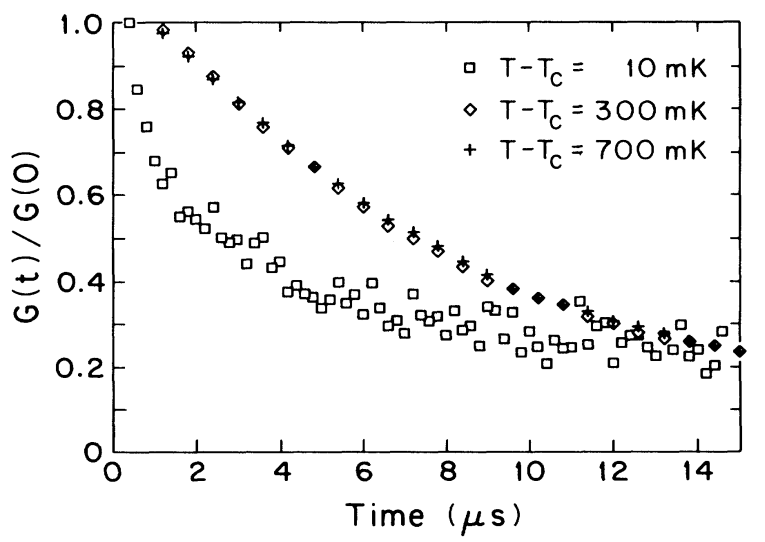

FIG. 2. The normalized $G(t)$ of IBW measured at different values of $T-T_{c}$. The measurements were made at $f=9 \mathrm{~Hz}$ and $\theta=90^{\circ}$. 
ponential function

$$
G(t)=G(0) \exp \left[-(t / \tau)^{\beta}\right],
$$

with $\tau$ and $\beta$ being fitting parameters. Figure 3 shows a typical correlation function $G(t)$ measured at $f=7.3 \mathrm{~Hz}$, $\theta=60^{\circ}$, and $T-T_{c}=13.5 \mathrm{mK}$. The solid line is a fit by Eq. (1) with $\beta=0.57$ and $\tau=5.3 \mu \mathrm{sec}$. To display the stretched exponential function more clearly, we also plot, in the inset to Fig. 3, the same $G(t)$ on the logarithmic scale as a function of $t^{\beta}$. This gives a straight line. From the fitting we find that the values of $\beta$ and $\tau$ decrease as the temperature approaches $T_{c}$, and that these parameters depend on the scattering angle. For example, when $\theta=90^{\circ}$ the value of $\beta$ changed from 0.76 at $T-T_{c}=55 \mathrm{mK}$ to $\sim 0.4$ at $T-T_{c}=10 \mathrm{mK}$. Whereas when $\theta=60^{\circ}$, the value of $\beta$ changed from 0.76 at $T-T_{c}=34 \mathrm{mK}$ to 0.57 at $T-T_{c}=13.5 \mathrm{mK}$. In the same parameter range the decay time $\tau$ varied from 9 to $3 \mu$ sec.

It has been shown ${ }^{8}$ that the correlation function $G(t)$ senses the velocity difference, $V(l)=v_{1}-v_{2}$, of pairs of points in the turbulent fluid separated by a distance $l$. If the turbulent fluid is seeded with small scattering particles, the motion of the seed particles Doppler shifts the scattered light. Therefore the phase of the detected light is modulated (due to frequency beating) at a frequency equal to the difference in Doppler shifts of all particle pairs in the scattering volume. In addition to these rapid phase fluctuations, there is a slow variation of the scattering amplitude from the turbulently stirred binary mixture. The correlation function $G(t)$ can be written as ${ }^{9}$

$G(t)=\int d \mathbf{r}_{1} \int d \mathbf{r}_{2}\left\langle\chi_{k}\left(S_{1}\right) \chi_{k}\left(S_{2}\right) \cos \left[k\left(v_{1}-v_{2}\right) t\right]\right\rangle$,

where the double integral runs over the scattering volume. In this equation a proportionality constant has

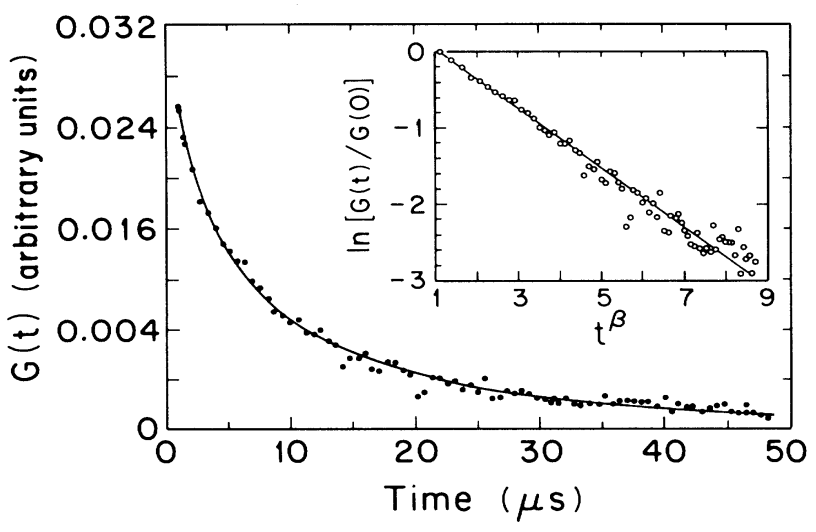

FIG. 3. A typical autocorrelation function $G(t)$ vs time $t$ for IBW mixture. The solid line is a fit by the stretched exponential function in Eq. (1). Inset: Semilog plot of the same $G(t)$ vs $t^{\beta}$. been omitted for simplicity. The angular brackets in Eq. (2) represent an ensemble average over the turbulent velocity field (hydrodynamic average). The two structure factors $\chi_{k}$, previously defined, depend on position through their dependence on the local shear $S(\mathbf{r})\left[S_{1} \equiv S\left(\mathbf{r}_{1}\right)\right]$. The positions $\mathbf{r}_{1}$ and $\mathbf{r}_{2}$ are displaced by $\left|\mathbf{r}_{1}-\mathbf{r}_{2}\right|=l \gg k^{-1}$. In the following we offer a tentative explanation for our observations in the turbulently stirred binary mixture.

Consider first the weak shear regime, where composition fluctuations are less affected by the turbulent velocity field. Because the decay of $G(t)$ due to the velocity fluctuations is much faster than the relaxation of composition fluctuations, these fluctuations can be treated as droplets which behave the same as the above-mentioned seed particles. Thus the structure factor $\chi_{k}$ becomes shear independent. Because the scattering volume has the form of a long, thin cylinder, the decay of $G(t)$ is controlled by its length rather than its diameter. Therefore $\chi_{k}$ can be factored out from the integral in Eq. (2), and the double integral in (2) can be simplified to a single integral, i.e.,

$$
G(t)=\left\langle\chi_{k}^{2}\right\rangle \int_{0}^{L} d l p(l)\langle\cos [k V(l) t]\rangle,
$$

where $p(l)=2(1-l / L) / L$ is the probability of finding a pair of scatterers separated by a distance $l$ in the scattering volume of length $L$. The structure factor $\chi_{k}$ is an average over the cross section of the beam. Tong et al. have used this technique to study turbulent pipe flow and grid flow. ${ }^{8}$ It was found that when the Reynolds number is large enough, $\langle\cos [k V(l) t]\rangle$ has an exponential form $\exp [-t / \tau(l)]$, with $\tau(l) \simeq[k u(l)]^{-1}$. If we take $\tau(l)=\tau_{0}(l / L)^{-1 / 3}\left\{\tau_{0} \simeq[k u(L)]^{-1}\right\}$, as in the Kolmogorov theory, we find that $G(t)$ is a scaling function of $k u(L) t^{8}$

In the strong shear region, the turbulent shear tends to destroy all the composition fluctuations with length scales larger than the characteristic length $\xi_{c}$, which is much shorter than $l_{d} \cdot{ }^{3}$ Since the shear is not uniform throughout the fluid, there will be only localized regions where the shear is large and composition fluctuations are destroyed. This causes the scattering to be momentarily attenuated in localized regions (of size $l_{d}$ or larger) throughout the fluid. We indeed see this spatial inhomogeneity in the scattering; the stirred mixture has a smoky appearance, even in the one-phase region. As a result the structure factor $\chi_{k}$ in Eq. (2) strongly depends on the local shear. Although not well justified, let us decouple $\left\langle\chi_{k}\left(S_{1}\right) \chi_{k}\left(S_{2}\right) \cos \left[k\left(v_{1}-v_{2}\right) t\right]\right\rangle$ in Eq. (2) as $\left\langle\chi_{k}\left(S_{1}\right) \chi_{k}\left(S_{2}\right)\right\rangle\left\langle\cos \left[k\left(v_{1}-v_{2}\right) t\right]\right\rangle$. Then we obtain

$$
G(t)=\int_{0}^{L} d l p(l) \Psi(l) \exp [-t / \tau(l)],
$$

where $\Psi(l)=\left\langle\chi_{k}\left(S_{1}\right) \chi_{k}\left(S_{2}\right)\right\rangle$ is the correlation of two structure factors, and the angular brackets represent an ensemble average over local shears. Here we have assumed that composition fluctuations do not react back to 
the turbulent velocity field. If we think of $\Psi(l)$ as being a probability density of encountering a region in the fluid with turbulent shear $S(l)=u(l) / l$, and we furthermore assume that $\Psi(l)$ is a Gaussian or exponential function of $S(l)$, we recover our results in weak shear regime, and also obtain a stretched exponential form of Eq. (1) for $G(t)$ when the shear is strong. We have not, however, been able to justify such a functional form for $\Psi(l)$.

In conclusion, when a binary liquid mixture is turbulently stirred, the temporal fluctuations in composition are dramatically changed as the temperature approaches $T_{c}$. This effect has been studied by photon correlation homodyne spectroscopy. In the weak shear region the measured $G(t)$ is independent of the temperature and has the same functional form as that seen in a simple turbulent fluid (water) seeded with small particles. In the strong shear region, $G(t)$ is found to be temperature dependent, and can be well fitted by a stretched exponential function of Eq. (1). We find that both the exponent $\beta$ and the decay time $\tau$ decrease as the temperature of the mixture approaches $T_{c}$, and that these parameters depend on scattering angle. The unusual behavior of $G(t)$ is presumably associated with the local suppression of the critical fluctuations produced by the vigorous stirring. Still lacking is a theory for the functional form of $G(t)$ in the strong shear regime.
We have benefitted from useful interactions with $R$. Piazza. J.S. is grateful to IBM for financial support. This work was supported in part by the National Science Foundation under Grant No. DMR-8611666.

(a) Present address: Department of Electronics, University of Pavia, Via Abbiateggraso, 27100 Pavia, Italy.

${ }^{1} \mathrm{H}$. E. Stanley, Introduction to Phase Transitions and Critical Phenomena (Oxford Univ. Press, New York, 1971).

${ }^{2}$ B. J. Berne and R. Pecora, Dynamic Light Scattering (Wiley, New York, 1976).

${ }^{3}$ A. Onuki, Phys. Lett. 101A, 286 (1984).

${ }^{4}$ A. Onuki and K. Kawasaki, Ann. Phys. (N.Y.) 121, 456 (1979).

${ }^{5}$ D. Beysens, M. Gbadamassi, and L. Boyer, Phys. Rev. Lett. 43, 1253 (1979).

${ }^{6}$ D. J. Pine, N. Easwar, J. V. Maher, and W. I. Goldburg, Phys. Rev. A 29, 308 (1984); C. K. Chan, W. I. Goldburg, and J. V. Maher, Phys. Rev. A 35, 1756 (1987).

${ }^{7}$ A. N. Kolmogorov, CR (Dokl.) Acad. Sci. URSS 30, 301; 31, 538 (1941); U. Frisch, P. Sulem, and M. Nelkin, J. Fluid Mech. 87, 719 (1978).

${ }^{8}$ P. Tong, W. I. Goldburg, C. K. Chan, and A. Sirivat, Phys. Rev. A 37, 2125 (1988); P. Tong and W. I. Goldburg, Phys. Fluids 31, 2841 (1988); A. Onuki, Phys. Lett. A 127, 143 (1988).

${ }^{9} \mathrm{~A}$. Onuki (unpublished). 